\title{
ON THE CARDINALITY OF A TOPOLOGICAL SPACE
}

\author{
ARTHUR CHARLESWORTH
}

\begin{abstract}
ABSTRACr. In recent papers, B. Sapirovskii, R. Pol, and R. E. Hodel have used a transfinite construction technique of Sapirovskii to provide a unified treatment of fundamental inequalities in the theory of cardinal functions. Sapirovskii's technique is used in this paper to establish an inequality whose countable version states that the continuum is an upper bound for the cardinality of any Lindelö space having countable pseudocharacter and a point-continuum separating open cover. In addition, the unified treatment is extended to include a recent theorem of Sapirovskii concerning the cardinality of $T_{3}$ spaces.
\end{abstract}

1. Introduction. Throughout this paper $\alpha$ and $\beta$ denote ordinals, $m$ and $\mathfrak{n}$ denote infinite cardinals, and $|A|$ denotes the cardinality of a set $A$. We let $d, c, L, w, \pi$, and $\psi$ denote the density, cellularity, Lindelöf degree, weight, $\pi$-weight, and pseudocharacter. (For definitions see Juhász [5].)

We also let $n w, \pi \chi$, and $p s w$ denote the net weight, $\pi$-character, and point separating weight. A collection $\Re$ of subsets of a topological space $X$ is a network for $X$ if whenever $p \in V$, with $V$ open in $X$, we have $p \in N \subseteq V$ for some $N$ in $\Re$. A collection $\mathscr{U}$ of nonempty open sets is a $\pi$-local base at $p$ if whenever $p \in V$, with $V$ open in $X$, we have $U \subseteq V$ for some $U$ in $\mathscr{U}$. A separating open cover $\mathcal{S}$ for $X$ is an open cover for $X$ having the property that for each distinct $x$ and $y$ in $X$ there is an $S$ in $\delta$ such that $x \in S$ and $y \notin S$. Now let $X$ be a topological space. Then by definition

$$
\begin{aligned}
& n w(X)=\kappa_{0} \cdot \min \{\mathfrak{n} \mid X \text { has a network of cardinality } \mathfrak{n}\}, \\
& \pi \chi(X)=\kappa_{0} \cdot \min \{\mathfrak{n} \mid \text { each point of } X \text { has a } \pi \text {-local base } \\
& \text { of cardinality at most } \mathfrak{n}\},
\end{aligned}
$$

and if $X$ is $T_{1}$, then

$$
\operatorname{psw}(X)=\aleph_{0} \cdot \min \{\mathfrak{n} \mid X \text { has a separating open cover } \mathcal{S}
$$

such that each point of $X$ is in at most $n$ members of $\delta$ \}.

We say that $X$ has a point-continuum separating open cover if and only if $\operatorname{psw}(X) \leqslant 2^{\omega}$.

In $\$ 2$ we use the technique of Śapirovskii to prove that $|X| \leqslant$ $\operatorname{psw}(X)^{L(X) \cdot \psi(X)}$ for any $T_{1}$ space $X$. The unified treatment of inequalities is

Received by the editors December 7, 1976 and, in revised form, March 15, 1977.

AMS (MOS) subject classifications (1970). Primary 54A25; Secondary 54D20.

Key words and phrases. Cardinality, Lindelö degree, pseudocharacter, separating open cover, cellularity, $\pi$-character, cardinal function.

- American Mathematical Society 1977 
extended in $\$ 4$ to include the recent theorem of Šapirovskii which states that $|X| \leqslant \pi \chi(X)^{c(X) \cdot \psi(X)}$ for any $T_{3}$ space $X$. A key step in our proof is based upon a property of the cellularity function discovered by Juhász; this property is used in $\$ 3$ to obtain a characterization of the cellularity suitable for our purposes.

The author is indebted to R. E. Hodel for many helpful comments. The preparation of this paper was supported by a grant from the University of Richmond.

2. A new upper bound for $|X|$. The transfinite construction technique used in the following proof is due to Šapirovskii [8]. The application of the Lindelöf degree was suggested by Remark 4.6 of Burke and Hodel [2].

THEOREM 2.1. If $X$ is $T_{1}$, then $n w(X) \leqslant p s w(X)^{L(X)}$.

Proof. Let $L(X)=\mathfrak{n}$ and let $\delta$ be a separating open cover for $X$ such that for each $x$ in $X$ we have $\left|\delta_{x}\right| \leqslant \mathfrak{m}$, where $\delta_{x}$ denotes the collection of members of $\mathcal{S}$ containing $x$.

We first show that $d(X) \leqslant \mathfrak{m}^{\mathfrak{n}}$. For each $\alpha<\mathfrak{n}^{+}$construct a subset $D_{\alpha}$ of $X$ such that

(1) $\left|D_{\alpha}\right| \leqslant \mathfrak{m}^{\mathrm{n}}$, and

(2) if $\mathscr{U}$ is a subcollection of $\cup\left\{\delta_{x} \mid x \in \cup_{\beta<\alpha} D_{\beta}\right\}$ such that $|\mathscr{Q}| \leqslant n$ and $X-\cup \mathcal{Q} \neq \varnothing$, then $D_{\alpha}-\cup \mathcal{\varnothing} \neq \varnothing$.

Such a $D_{\alpha}$ can be constructed since the number of possible $U$ 's at the $\alpha$ th stage of construction is $\leqslant\left(\mathfrak{m}^{\mathfrak{n}} \cdot \mathfrak{n} \cdot \mathfrak{m}\right)^{\mathfrak{n}}=\mathfrak{m}^{\mathfrak{n}}$. Let $D=\cup_{\alpha<\mathfrak{n}^{+}} D_{\alpha}$. Clearly $|D| \leqslant \mathfrak{m}^{\mathfrak{n}}$. Furthermore, if there is a point $p$ in $X-\bar{D}$, then one can use $L(\bar{D}) \leqslant \mathfrak{n}$ to select a subcollection $\mathscr{U}$ of $\cup\left\{\mathcal{S}_{x} \mid x \in D\right\}$ such that $|\mathscr{}| \leqslant \mathfrak{n}$, $p \in X-\cup \mathcal{U}$, and $D \subseteq \cup \mathscr{Q}$; this contradicts (2).

Since $d(X) \leqslant \mathfrak{m}^{\mathfrak{n}}$ we know that $|\mathcal{S}| \leqslant \mathfrak{m}^{n}$. Let $\mathscr{\Re}=\{X-S \mid S$ is the union of at most $n$ members of $\delta\}$. Then $|\Re| \leqslant \mathfrak{m}^{\mathfrak{n}}$ and $\Re$ is a network for $X$.

LEMMA 2.2. If $X$ is $T_{1}$, then $|X| \leqslant n w(X)^{\psi(X)}$.

Proof. Let $n w(X)=m$ and $\psi(X)=n$ and let $\Re$ be a network for $X$ such that $|\Re| \leqslant m$. Then

$$
\{\{x\} \mid x \in X\} \subseteq\left\{\cap \Re^{\prime}\left|\Re^{\prime} \subseteq \Re,\right| \Re^{\prime} \mid \leqslant \mathfrak{n}\right\} \text { so }|X| \leqslant \mathfrak{m}^{n} .
$$

Theorem 2.3. If $X$ is $T_{1}$, then $|X| \leqslant p s w(X)^{L(X) \cdot \psi(X)}$.

Proof. $|X| \leqslant n w(X)^{\psi(X)} \leqslant\left(p s w(X)^{L(X)}\right)^{\psi(X)} \leqslant p s w(X)^{L(X) \cdot \psi(X)}$.

COROLlaRY 2.4. If $X$ is a Lindelöf space with countable pseudocharacter and having a point-continuum separating open cover, then $|X| \leqslant 2^{\kappa_{0}}$.

REMARK 2.5. Let $X$ be the product of $2^{\boldsymbol{x}_{0}}$ copies of the two point discrete space. Then $X$ is Lindelöf and $w(X)=2^{\kappa_{0}}$ but $|X|>2^{\kappa_{0}}$. This shows the importance of countable pseudocharacter in Corollary 2.4 .

Remark 2.6. Corollary 2.4 provides a partial solution to Problem 5.2 of 
Arhangel'skii [1] concerning whether the continuum is an upper bound for the cardinality of a Hausdorff Lindelöf space having countable pseudocharacter.

3. A characterization of the cellularity. In this section we show that the cellularity can be characterized by a Lindelöf-like property. This characterization is based on the following lemma.

LEMMA 3.1 (JuHÁsz [6]). Let $X$ be a topological space. If $c(X) \leqslant n$ and $\mathcal{Q}$ is an open cover of $X$, then $\mathcal{Q}$ has a subcollection $\mathcal{V}$ of cardinality $\leqslant \mathfrak{n}$ such that $\cup \mathfrak{V}$ is dense in $X$.

THEOREM 3.2. Let $X$ be a topological space. The following statements are equivalent.

(1) $c(X) \leqslant n$.

(2) Every collection of open sets whose union is dense in $X$ has a subcollection of cardinality $\leqslant \mathrm{n}$ whose union is dense in $X$.

(3) Every collection $\mathscr{U}$ of open sets in $X$ has a subcollection $\mathcal{V}$ of cardinality $\leqslant n$ such that $\cup \mathscr{Q} \subseteq \bar{\cup}$.

Proof. (1) implies (2). Let $\mathscr{U}$ be a collection of open sets such that $\cup \mathcal{Q}=D$ and $\bar{D}=X$. Since $c(X) \leqslant \mathrm{n}$ we have $c(D) \leqslant \mathfrak{n}$ so by Lemma 3.1 we may select a subcollection $\mathcal{V}$ of $\mathscr{U}$ of cardinality $\leqslant \mathfrak{n}$ such that $\cup \mathcal{V}$ is dense in $D$. Since $\cup \mathcal{V}$ is dense in $X$, we are done.

(2) implies (1). Suppose $c(X)>\mathfrak{n}$ and let $\mathscr{U}$ be a maximal collection of $>\mathfrak{n}$ pairwise disjoint open sets. Then the union of $\mathscr{U}$ is dense in $X$ but there cannot be a subcollection of $\mathscr{Q}$ of cardinality $\leqslant \mathfrak{n}$ whose union is dense in $X$.

(1) implies (3). Let $\mathcal{U}$ be a collection of open sets and let $Y=\cup \mathcal{U}$. Since $c(X) \leqslant \mathfrak{n}$ and $Y$ is open we have $c(Y) \leqslant \mathfrak{n}$ so by Lemma 3.1 we may select a subcollection $\mathcal{V}$ of $\mathscr{U}$ such that $|\mathscr{V}| \leqslant n$ and $Y \subseteq \bar{U} \mathcal{V}$.

(3) implies (1). See proof of (2) implies (1).

REMARK 3.3. By considering the dual of (2) in Theorem 3.2, one can rewrite the topological form of Martin's Axiom [5, p. 58] as follows. If $X$ is a compact Hausdorff space having the property that each family of closed sets whose intersection is nowhere dense includes a countable subfamily whose intersection is nowhere dense, then $X$ cannot be written as the union of less than $2^{\kappa_{0}}$ nowhere dense sets.

4. The upper bound of Sapirovskii. Theorem 3.2 insures that the full strength of the cellularity is used in the following proof. Also note the application of Sapirovskiī's construction technique.

THEOREM 4.1. If $X$ is regular, then $d(X) \leqslant \pi \chi(X)^{c(X)}$.

Proof. Let $\pi \chi(X)=m$ and $c(X)=n$. For each $x$ in $X$ let $\mathscr{B}_{x}$ be a $\pi$-local base for $x$ such that $\left|\mathscr{B}_{x}\right| \leqslant \mathrm{m}$. Construct for each $\alpha<\mathfrak{n}^{+}$a subset $D_{\alpha}$ of $X$ such that

(1) $\left|D_{\alpha}\right| \leqslant \mathfrak{m}^{\mathfrak{n}}$, and

(2) if $\mathscr{U}$ is a subcollection of $\cup\left\{\mathscr{B}_{x} \mid x \in \cup_{\beta<\alpha} D_{\beta}\right\}$ such that $|\mathscr{U}| \leqslant n$ 
and $\bar{x}-\overline{\cup \mathscr{U}} \neq \varnothing$, then

$$
D_{\alpha}-\overline{\cup थ} \neq \varnothing
$$

Let $D=\cup_{\alpha<1^{+}} D_{\alpha}$. Clearly $|D| \leqslant \mathfrak{m}^{\mathrm{n}}$. To see that $D$ is dense in $X$, suppose otherwise and let $V$ be a nonempty open set such that $D \cap \bar{V}=\varnothing$. For each $x$ in $D$ let $U_{x}$ be the union of all those members of $\mathscr{B}_{x}$ which miss $V$. Note that $D \subseteq \overline{\cup_{x \in D} U_{x}}$. By Theorem 3.2 there is a subset $E$ of $D$ such that $|E| \leqslant \mathfrak{n}$ and $\cup_{x \in D} U_{x} \subseteq \bar{U}$, where $\mathscr{U}=\left\{U_{x} \mid x \in E\right\}$. Let $\alpha<\mathfrak{n}^{+}$ be such that $E \subseteq \cup_{\beta<\alpha} D_{\beta}$. Since $V \subseteq X-\cup \mathcal{Q}$ we have $X-\overline{\cup \mathcal{Q}} \neq \varnothing$ so $D_{\alpha}-\overline{\cup \mathscr{Q}} \neq \varnothing$ by (2); but this contradicts the fact that $D \subseteq \bar{\cup}$.

The following example, contributed by E. K. van Douwen, shows that "regular" cannot be replaced by "Hausdorff" in Theorem 4.1.

EXAmple 4.2 (VAn Douwen). There is a Hausdorff space $X$ such that $\chi(X)=2^{\mathrm{n}}, c(X)=\aleph_{0}$, and $d(X)>2^{\mathrm{n}}$.

To construct the example, let $X$ be the product of $2^{\text {n }}$ copies of the two point discrete space and let the topology on $X$ be defined as follows. Choose a bijection $\varphi$ from $X$ to the set $2^{2^{n}}$ and for each subset $U$ of $X$ and each point $x$ of $X$ let $U[x]=\{y \in U \mid \varphi(x) \leqslant \varphi(y)\}$; then basic open sets are to be of the form $U[x]$, where $x \in X$ and $U$ is open in the product topology on $X$.

Clearly $\chi(X)=2^{n}$ and $d(X) \geqslant c f\left(2^{2^{n}}\right)>2^{n}$. To see that $c(X)=\aleph_{0}$, suppose that $\left\{U_{\alpha}\left[x_{\alpha}\right] \mid \alpha<\aleph_{1}\right\}$ is a pairwise disjoint collection. Since each $U_{\alpha}$ is open in the product topology (whose cellularity is countable), there are distinct $\alpha$ and $\beta$ such that $U_{\alpha} \cap U_{\beta} \neq \varnothing$. Since $\left|U_{\alpha} \cap U_{\beta}\right|=2^{2^{n}}$, there is a $y$ in $U_{\alpha} \cap U_{\beta}$ such that $\varphi\left(x_{\alpha}\right) \leqslant \varphi(y)$ and $\varphi\left(x_{\beta}\right) \leqslant \varphi(y)$; thus $y \in U_{\alpha}\left[x_{\alpha}\right] \cap$ $U_{\beta}\left[x_{\beta}\right]$, a contradiction.

LEMMA 4.3 (EFimov [3]). If $X$ is regular, then $w(X) \leqslant \pi(X)^{c(X)}$.

THEOREM 4.4 (ŠAPIROVSKII [9]). If $X$ is regular, then $w(X) \leqslant \pi \chi(X)^{c(X)}$.

Proof. Since $\pi(X) \leqslant d(X) \cdot \pi \chi(X)$ we have $w(X) \leqslant \pi(X)^{c(X)} \leqslant(d(X)$. $\pi \chi(X))^{c(X)}<d(X)^{c(X)} \cdot \pi \chi(X)^{c(X)} \leqslant \pi \chi(X)^{c(X)}$.

THEOREM 4.5 (Š́ APIROVSKIİ [9]). If $X$ is $T_{3}$, then $|X| \leqslant \pi \chi(X)^{c(X) \cdot \psi(X)}$.

Proof. Applying Lemma 2.2 gives

$$
|X| \leqslant n w(X)^{\psi(X)} \leqslant w(X)^{\psi(X)} \leqslant \pi \chi(X)^{c(X) \cdot \psi(X)} .
$$

\section{REFERENCES}

1. A. V. Arhangel'skii, On cardinal invariants, General Topology and Its Relations to Modern Analysis and Algebra IV, Academic Press, New York, 1972, pp. 37-46.

2. D. K. Burke and R. E. Hodel, On the number of compact subsets of a topological space, Proc. Amer. Math. Soc. 58 (1976), 363-368.

3. B. Efimov, Extremally disconnected bicompacta of continuum $\pi$-weight, Soviet Math. Dokl. 9 (1968), 1404-1407.

4. R. E. Hodel, New proof of a theorem of Hajnal and Juhasz on the cardinality of topological spaces, Bull. Acad. Polon. Sci. Sér. Sci. Math. Astromom. Phys. 24 (1976), 999-1000.

5. I. Juhász, Cardinal functions in topology, Math. Centrum, Amsterdam, 1971. 
6. I. Juhász, On two problems of A. V. Arhangel'skii, General Topology and Appl. 2 (1972), 151-156.

7. R. Pol, Short proofs of two theorems on cardinality of topological spaces, Bull. Acad. Polon. Sci. Sér. Sci. Math. Astronom. Phys. 22 (1974), 1245-1249.

8. B. Sapirovskii, Discrete subspaces of topological spaces. Weight, tightness, and Souslin mumber, Dokl. Akad. Nauk SSSR 202 (1972), 779-782. (Russian) MR 45 \# 1100.

9. , Canonical sets and character. Density and weight in compact spaces, Soviet Math. Dokl. 15 (1974), 1282-1287.

Department of Mathematics, University of Richmond, Richmond, Virginia 23173 